\title{
Design and Simulation of the Novel Wireless Sensor Network Spectrum Allocation Algorithm
}

\author{
Wenzhun Huang ${ }^{\mathrm{a}^{*}}$, Xinxin Xie ${ }^{\mathrm{b}}$ and Hui Zhang ${ }^{\mathrm{c}}$ \\ ${ }^{1}$ School of Information Engineering, Xijing University, Xi'an 710123, China \\ ahuangwenzhun@xijing.edu.cn, b346148500@qq.com, ${ }^{\text {c}}$ zhang_hui@xijing.edu.cn
}

\begin{abstract}
Keywords: Wireless Sensor Network, Spectrum Allocation, Simulation, Implementation, Novel. Abstract. In this paper, we conduct research on the design and simulation of the novel wireless sensor network spectrum allocation algorithm. Cognitive radio technology is able to perceive the general surrounding environment spectrum, spectrum hole detection, the use of artificial intelligence from the environment, learning, and real-time change working parameters of the system, and make use of the "secondary" of the spectrum, thus improve the spectrum utilization. How to effectively implement frequency spectrum resource management has become the key to realize the reliable communication. Through the improvement, the distribution of the weight before sent to the fusion center is completed, and according to the set threshold values, for each of the cognitive users respectively, then the verdict to the fusion center, this is the fusion center receives each user of the verdict. In the experiment part, we simulate the propose method and verify the robustness and feasibility of our method.
\end{abstract}

\section{Introduction}

Wireless sensor network consists of wireless communication ability, awareness and capacity of micro sensor nodes, self-organizing them into a network, and communication in the form of multiple hops between nodes. Application technology from sensor hardware device and platform manufacturing, and network application technology development of the angle sensor network to the real application transformation, the network support technology including positioning technology, synchronization technology, general security technology, network information processing technology, etc., to network performance as main goal of ascension improves overall quality of service and intelligent network.

The network in data transmission at the same time, also to deal with data by data acquisition node to user domain transfer to complete the procedure of dealing with complex and complete information, while specific information processing methods according to different application requirements for the design and implementation that could be summarized as the listed aspects. (1) Reduce transmission delay. Transmission delay is very important in many applications of network indicators, in the process of transmission of the data processing methods, such as sorting, compression, can decrease the rate of transmission congestion that reduce the data in the network transmission delay. (2) To reduce the data redundancy. In sensor networks, location close to the sensor nodes to the surrounding environment of information collected, tend to have high similarity, repeat send redundant information is clearly not the right thing to do and therefore, it will have high correlation of the multiple sensor nodes to collect information merging processing first. (3) Improve the data accuracy. Network information processing extends the individual perception data connectivity in the district, the work mode, to work through information interaction between nodes, ability of the mathematical calculation, network management, mobility management, business management, data transmission, such as the optimization results, the business operation of auxiliary upper, transfer, routing and user decision, etc.

To deal with the mentioned challenges, in this paper we conduct research on the design and basic simulation of the novel wireless sensor network spectrum allocation algorithm. By high frequency spectrum utilization technology, first of all need to rediscover spectrum, spectrum is not specific and limited resources, it is abstract and unlimited resources, its utilization ratio depends on the technology used. Second, to be detailed discussed efficient spectrum utilization technology can make full use of the spectrum and the spectrum sharing technology without changing the existing spectrum allocation under the overall structure, through different radio spectrum sharing system to improve the spectrum 
efficiency, implementation difficulty relatively dynamic spectrum allocation strategy is small as the coexist in sharing all spectrum method, the method of frequency spectrum sharing in the actual use of the most successful, this basically is it simple implementation, and without using the cognitive radio spectrum perception. The application scenarios is shown in Fig. 1[1-3].

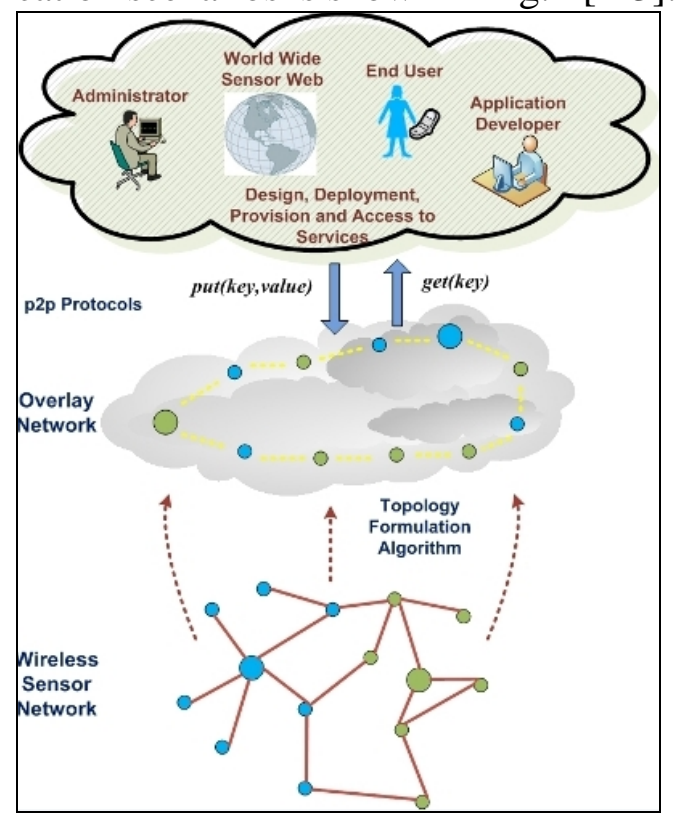

Fig. 1. The General Application Scenarios of the Wireless Sensor Network

\section{The Proposed Methodology}

The Wireless Sensor Network (WSN) and the Characteristics. Sensor network applications have the feature of data flow driven, so we in the dynamic testing sensor network application often need to track the execution of the program into interval, contains the error range of program behavior through the testing to find errors in the program.

In the typical application of wireless sensor network, sensor nodes are used to perceive physical parameters in a certain area or phenomenon. After the sensor acquisition task, usually to send data to the information fusion and processing centers are extracted from a distance. Sensor network is not just a sense of the data acquisition and the transparent transmission, the network in real time and reliably transmit data at the same time, is also an information processing factories, on the basis of the original data collection, internal work for a large amount of the data processing, network transmission to the network of the external terminal will sensory information from the mass that is likely to be messy and difficult to understand the original data of extraction and derived for some specific user valuable and meaningful data after processing [4-5].

Generally, the applications of the WSN from the data processing level could be organized as the follows. (1) Characteristics of SBR process. On a particular mode of a set of basic measurements to calculate and transform, highlight the typical characteristics of this model, according to the different characteristics of data for data processing. The same analog sources have different feature extraction methods to choose according to different application scenarios need to extract the characteristics. (2) Level fusion processing. The purpose of correlation analysis is to find out hidden in the data network. Some cases the perception of information acquisition as well as the single data cannot represent the characteristics of the object, as need more data said together and the results of the data correlation the credible degree of the association rules. (3) Said level processing. According to the different needs of the users, the final data presentation may be related to the data structure of data transmission network inside then we need to reconstruct the data. To extract the data structure of the network description, according to the needs of users, through the corresponding mapping function to conversion of the data structure, so that users can access to the correct data information. 
The Spectrum Hole Feature Analysis. In the cognitive radio environment, authorized users have absolute rights to spectrum, cognitive users can only access channel. So in this paper, considering the channel model is the authorized user probability is low, the emergence of the cognitive users can in the current available channel, choose to suit the own channel access.

Besides the authorized user arrival rate and channel SNR, and also gives the empty occupancy rate can reflect the cognitive radio technology characteristics and the switching rate, due to the authorized users use spectrum hole length is different, through statistics of historical information, information given spectrum hole on each channel. Factor is the greatest influence on cognitive users to authorized users, cognitive users detect authorized users when they arrive, as must immediately or have to wait in line to switch to other channel available spectrum, so the arrival rate of authorized users do feature analysis is critical. Correspondingly, the probability could be expressed as the follows.

$$
P=[N \times p] / \mathrm{T}
$$

Where the $p$ represents the probability of a single authorized service user requests, Tdenotes the authorized users request service expectations. In order to describe the spectrum holes in the history of the utilization, this paper statistics the spectrum hole occupancy probability, the channel model on the basis of the history of the spectrum hole usage statistics, the information as the evaluation index of the spectrum holes that is able to reflect the degree of use of the channel. Switching rate spectrum can reflect the stability of the spectrum hole, on the choice of the frequency spectrum hole, the greater the stability of the cognitive user's choice of spectrum hole, provide the better the quality of service and frequent switching can cause unnecessary overhead, adds unnecessary burden to cognitive users. To evaluate the efficiency, we introduce the switching parameter as the formula 2.

$$
\chi=N / T
$$

In describing the characteristics of the spectrum hole, there are many other factors to consider, such as the free frequency bandwidth, transmission power spectrum hole current status, channel, etc. The general demonstration of the spectrum hole feature is shown in Fig. 2. Given in this paper, the spectrum hole prediction mechanism research is still in the early stage, and some influence factors not included in the investigation.

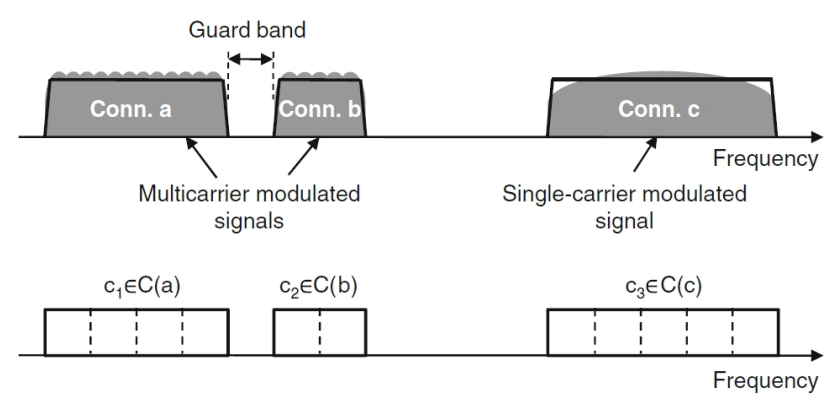

Fig. 2. The General Demonstration of the Spectrum Hole Feature

The Novel Spectrum Allocation Algorithm. Various cognitive users random distribution inside the village in a network, on one's own perception the main network spectrum usage, through public control channel transmission spectrum using information between each other, and then on the mutually agreed good channel for communication links. Due to the characteristics of game theory is the game players through their mastery of the information available to choose their own strategy, it is the characteristics of the distributed network. At the beginning of each time slot, all nodes to check whether there is data transfer: if not, the network within the time slots to enter a core dormant state; Otherwise, the network began to test spectrum. A significant feature of wireless sensor network is have a large number of dense distribution of sensor nodes, cooperate this topology is very suitable for spectrum detection that could be expressed as the follows [6-7].

$$
E_{\text {general }}=\sum_{i=1}^{N_{s}} e_{s}+\frac{M_{i}}{M} \cdot e_{\max }
$$

We can put the cognitive radio and dynamic spectrum allocation problem with the game process is described. In the process of the game, players are cognitive users and their action is the selection of the 
transmission channel, and their associated utility and quality of the selected channel. The game theory model of the general form of spectrum allocation problem can be expressed as follows.

$$
G T=\left\{N,\left\{S_{i}\right\}_{i \in N},\left\{S_{j}\right\}_{j \in N}\right\}
$$

Level difference is refers to the general cognitive user preferences of different spectrum, spectrum characteristics, that is, even if is the same, cannot replace each other and the vertical difference of the spectrum refers to the frequency spectrum of quality differences, such as free condition and state of the channel capacity and perception, the spectrum of the free condition and channel capacity through cognitive users receive SNR. In order to make full use of idle spectrum resource and without causing excessive interference to primary users network usually require a secondary user network detection reliability to satisfy the following regulation [8-9].

$$
P_{f a s} \leq \sigma ; P_{d s} \geq \varepsilon
$$

According to the principle of Nash equilibrium, if none of the participants to act on its own changes to improve their income, the action is participants set the corresponding vector is called the Nash equilibrium. If meet the following conditions, this paper qualitative judgment Nash equilibrium game process can save. (1) Cognitive users set are limited. (2) The utility function is in action on the space of continuous concave function. (3) Cognitive user policy set is closed and bounded convex set.

In a known state transition probability of the main user channel case, consider in each time slot on which channel detection can make the minimum number of questions partially observable Markov decision process can be used to solve. In the Markov decision process, all the better information for decision making can be included in the prior probability of the system state vector.

\section{Experiment and Verification}

Simulation experiment, the algorithm in this section for general performance analysis of this kind of cooperative detection strategy without having to specify the specific detection methods, energy detection, matched filter detection or smooth circulation features, assuming constant correct detection probability of each sensor node. The Fig. 3 demonstrates the verification result.

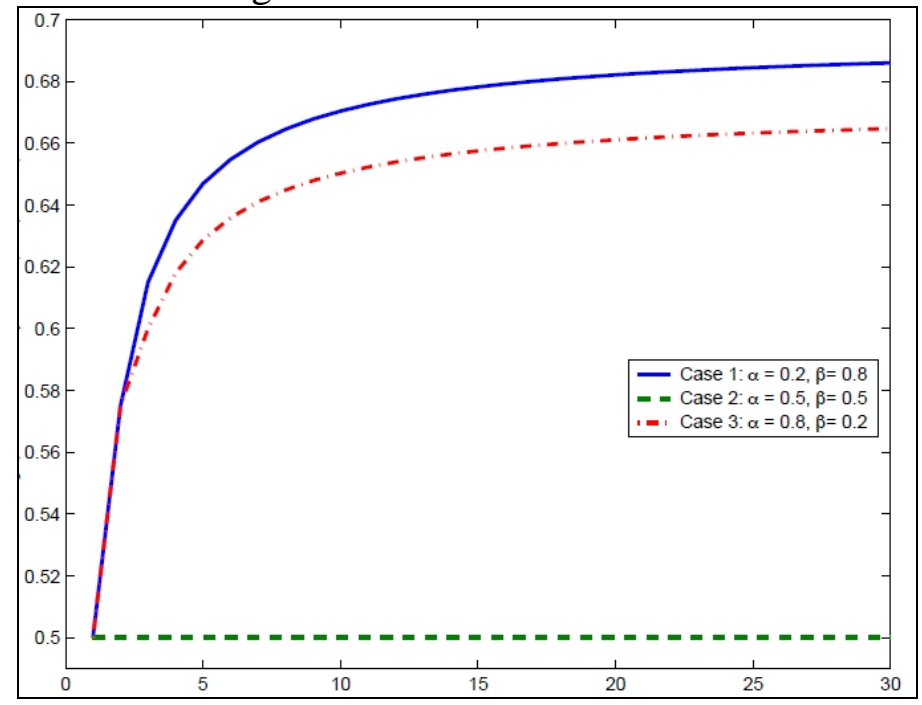

Fig. 3. The Simulation of the Novel Wireless Sensor Network Spectrum Allocation Algorithm

Fig. 3 shows the user cognitive reaction function curve, and the curve of intersection is the Nash equilibrium that can be seen from the diagram, each cognitive users is the best response to another cognitive strategies on the point of linear function. Nodes share a good detection performance, the total number of nodes respectively take 5 and 25 , credibility voting algorithm is relatively large save energy is constant, the false alarm probability is always less than voting algorithm of large number, and the detection probability were little changed. Total number of the testing nodes must, detection performance good nodes increases, the proportion of the confidence vote algorithm save energy linear decrease relatively, the false-alarm probability and detection probability voting algorithm to improve 
the level of large number decreases relatively. Thus credibility voting algorithm in the transmission test results of node proportion always approximation approach in good detection performance node, the proportion of energy saving is the part of the node detection performance is the poor relative proportion. And when the proportion of detection performance good nodes is low by node detection performance difference is serious. Sensing to shorten the sensor nodes sleep time, increase the burden of the nodes to monitor channel for the exchange of test results and traffic. Inevitable detect errors and will cause the sensor network conflict with major customers, further increase additional consumption.

\section{Conclusion}

In this paper, we conduct research on the design and simulation of the novel wireless sensor network spectrum allocation algorithm. Sensor network one of the important supporting technologies for time synchronization technology, time synchronization service in the sleep mechanism, data fusion, time division multiple access, and the other applications. Many location, safety, and tracking protocol also require a timestamp mark node of sensory information. For design of energy efficient wireless sensor network (WSN), the time synchronization is an important research content. Cognitive radio spectrum allocation problem is a NP problem, essentially solve for the optimal solution of this problem is very complicated and difficult to achieve. Therefore we propose the novel algorithm to enhance traditional methods with experimental verification.

\section{Acknowledgements}

This research was financially supported by industrial science and technology research plan in Shaanxi Province of China (Program No. 2016GY-141).

\section{References}

[1] H. J. Visser and R. J. M Vullers: Proceedings of the IEEE Vol.101.6 (2013), p.1410.

[2] T. Tom, S. Tom, B. Steven and S. Juan: IEEE Sensors Journal Vol.13.3 (2013), p.909.

[3] M. Bhardwaj, D. K. Kotary and S. Soni: Special Issue of International Journal of Computer Applications on Wireless Communication and Mobile Networks (2012), p.65.

[4] K. S. Yildirim, and Önder Gürcan: IEEE Transactions on Wireless Communications Vol. 13.7 (2014), p.3650.

[5] D. L. Ndzi, L. M. Kamarudin, A. A. Muhammad Ezanuddin: Progress In Electromagnetics Research B Vol. 36 (2012), p.283.

[6] Y. K. Joshi and Marwan Younis: Global Communications Conference (GLOBECOM), 2012 IEEE (2012), p.652.

[7] Fuu-Cheng Jiang, Der-Chen Huang, Chao-Tung Yang and Fang-Yi Leu: The Journal of Supercomputing Vol.59.3 (2012), p.1312.

[8] K. N. Qureshi and A. H. Abdullah: World Applied Sciences Journal Vol.30.10 (2014), p.1218.

[9] A. K. M. Muzahidul Islam, K. Wada and Wei Chen: International Journal of Innovative Computing, Information and Control (IJICIC) Vol.9 (10) (2013), p.4085. 